\title{
DEM based Terrain Sketch Mapping in a Loess Landform By Considering visual hierarchy and feature generalization
}

\author{
考虑视觉层次和要素综合下的基于 DEM 的黄土地貌地形素描图 \\ Yihan Cheng ${ }^{\mathrm{b}}$, Xin Yang ${ }^{\mathrm{a},}$, Hailong Liu ${ }^{\mathrm{c}}$
}

${ }^{a}$ Key Laboratory of Virtual Geographic Environment, Ministry of Education, Nanjing Normal University,Nanjing 210023, China

${ }^{b}$ State Key Laboratory Cultivation Base of Geographical Environment Evolution, Nanjing 210023, China; YHCheng1029@163.com

${ }^{c}$ Jiangsu Center for Collaborative Innovation in Geographical Information Resource Development and Application, Nanjing 210023, China; 171302080 @ stu.njnu.edu.cn (H.L.)

* Correspondence: xxinyang@njnu.edu.cn; Tel.: +86-136-4518-5065

Keywords: terrain sketch map, DEM, loess landform, visual hierarchy division, visual outline generalisation

\section{Abstract:}

A terrain sketch map can effectively describe the terrain features and spatial pattern of a landscape, which are sketched with lines by a geographer after recognizing, analyzing and summarizing the features of a terrain scene to present his/her understanding of a landscape (Bao 1956, Chen 1958, Liu 1988). Compared with a digital elevation model (DEM) scene map, a terrain sketch map, as a scientific abstraction and expression of geomorphological features from the perspective view, is characterized by conciseness and profoundness to show the intrinsic features of a landscape. With the rapid development of computer visualization and digital terrain analysis methods, computer-assisted terrain sketches based on DEM may be automatically generated, depending on geographic cognition theory and the sketch description method.

Previous research has generated terrain scene maps based on DEM data and has produced various styles of terrain maps, i.e. oil painting, ink painting, pen and pencil sketch maps (Visvalingam et al. 1998, Whelan et al. 2003, Way et al. 2006, Bhattacharjee et al. 2008). However, those computer-drawn sketch maps have focused on the artistic effect rather than depicting terrain features and landform structures. Moreover, the area used for sketch mapping in those researches was relatively small, and visual hierarchy was not considered. Thus, those maps differ considerably from hand-drawn sketch maps by geographers or geologists. Therefore, this paper aims to generate terrain sketch map which can express terrain features as well as artistic effect.

In this study, one loess ridge-hill area (Wuqi) in the loess plateau of China was selected by considering the typicality of the geomorphic types and the availability of data. The $5 \mathrm{~m}$ resolution DEM data was obtained from the National Administration of Surveying, Mapping and Geoinformation, with an area of $102 \mathrm{Km}^{2}$.

Three aspects as terrain features, visual hierarchy and feature generalization were considered in this paper.

Firstly, the typical terrain features must be presented in the sketch map. The loess shoulder line, bank gully lines and surface flow lines in loess landform area were extracted (Yang et al. 2017, Yang et al. 2018). Then based on the Bresenham algorithm (Bresenham 1965), the visual outlines in perspective view were generated.

Secondly, the visual hierarchy is considered in this study. In a terrain sketch map, more topographically-detailed information is described in the near view, whereas only the approximate outlines and less topographical information are displayed in the distant view. Therefore, a self-adapted visual hierarchy division method was proposed. The scenes are visually divided into three levels, i.e. near view, medium view and distant view. Different numbers and types of terrain features are assigned to each visual level to obtain the effect of clear near and blurred distant views.

Thirdly, in complexity terrain area, the scene will not only look staggered and messy, but will also affect topographic recognition even only visual outlines are displayed in distant view. Therefore a method of combining DEM and visual watershed generalization was proposed to simplify the visual outlines in distant view.

Finally, the terrain sketch map is assessed and verified through a questionnaire survey which includes three specialist experts, seven graduate students and twelve undergraduate students with knowledge in geomorphology or geography.

The result show that the important feature lines added to the terrain sketch map could effectively help to recognize the typical landform characteristics in the loess plateau of China. The severity of soil erosion by gullies are obviuosly presented (Fig.1d) comparing to the sketch mapping with only visual outlines (Fig1b). The map look messy and unsigtly if all the terrain feature lines were put into the map (Fig.1c). By considering the law of air perspective, the visual hierarchy was divided and the visual outlines in the distant view was generalized, thereby realizing the characteristics of a terrain sketch map clear in near view and blurred in distant view (Fig.1d). It also showed from the questionnaire that DEM resolution influence the performance of our method especially with coarse resolution DEM. The experts suggested that filter noise in near view and generalisation in distant view can be further improved. 


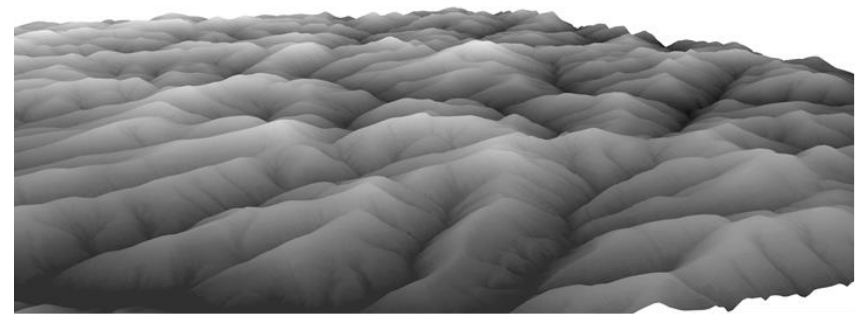

a. DEM in 3D view

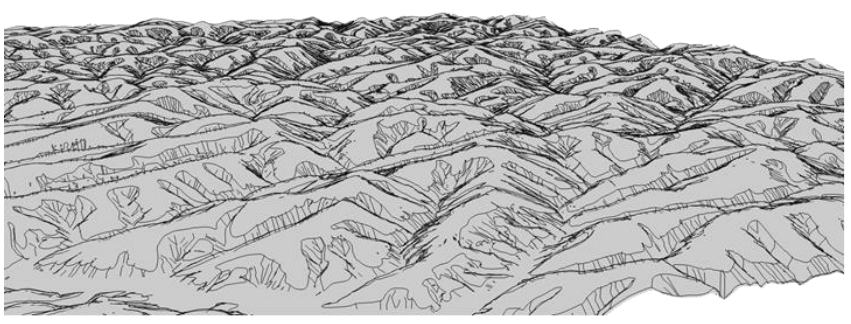

c. sketch map by terrain feature lines

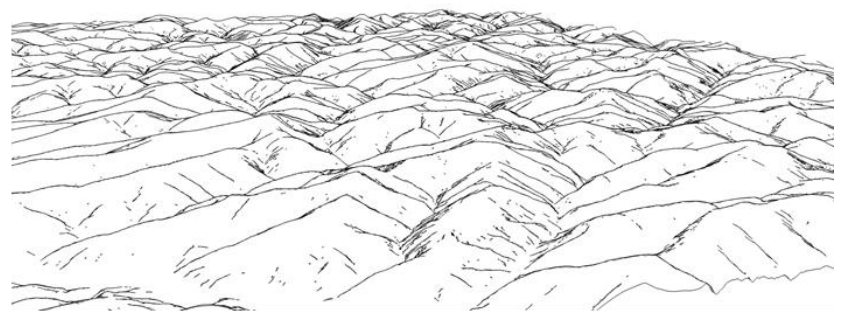

b. sketch map by visual outlines

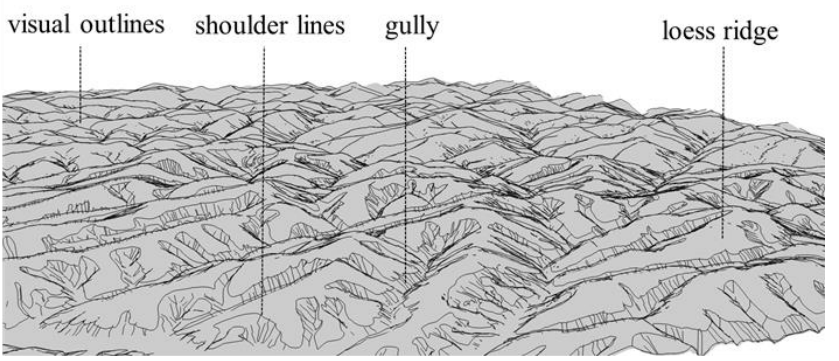

d. sketch map with visual hierarchy and feature generalization

Figure 1 DEM and terrain sketch maps

\section{Reference}

Bao, L.W.J.; 1956. Geography and Sketch. Beijing, China: People's Education Press (In Chinese).

Bhattacharjee, S. and P. Narayanan., 2008. Real-time painterly rendering of terrains. Computer Vision, Graphics \& Image Processing, 2008. ICVGIP'08. Sixth Indian Conference on, IEEE.

Bresenham, J. E. (1965). "Algorithm for computer control of a digital plotter." IBM Systems journal 4(1): 25-30.

Chen, S.P.; 1958. Sketch method of landscape. Beijing, China: Geological Publishing House (In Chinese).

Liu, J.; 1988. Geographic drawing. Xi'an, China: Shaanxi Normal University Press (In Chinese).

Visvalingam, M. and K. Dowson (1998). "Algorithms for sketching surfaces." Computers \& Graphics 22(2-3): 269280.

Way, D.-L. and Z.-C. Shih (2006). "Wrinkle rendering of terrain models in Chinese landscape painting." IEICE TRANSACTIONS on Information and Systems 89(3): 1238-1248.

Whelan, J. C. and M. Visvalingam (2003). Formulated silhouettes for sketching terrain. Theory and Practice of Computer Graphics, 2003. Proceedings, IEEE.

Yang, X., M. Li, J. Na and K. Liu (2017). "Gully boundary extraction based on multidirectional hill - shading from high - resolution DEMs." Transactions in GIS 21(6): 1204-1216.

Yang, X., J. Na, G. Tang, T. Wang and A. Zhu (2018). "Bank gully extraction from DEMs utilizing the geomorphologic features of a loess hilly area in China." Frontiers of Earth Science: 1-18. 